\title{
Turbo-Coded Star-QAM for Cooperative Wireless and Optical-Fiber Communications
}

\author{
Dandan Liang, Xinyi Xu, Soon Xin Ng and Lajos Hanzo \\ Communications, Signal Processing and Control Group \\ University of Southampton \\ Southampton, SO17 1BJ, U.K. \\ Email: $\{$ dl4e08,xx08r,sxn,lh\}@ecs.soton.ac.uk
}

\begin{abstract}
A low-complexity cooperative wireless and optical-fiber communication scheme is proposed for uplink communication in a Fractional Frequency Reuse (FFR) based multicell, multiuser system. The FFR principle is invoked for improving the cell-edge performance without reducing the throughput of the cell-center. Each cell is illuminated with the aid of six Remote Antennas (RAs), which are connected to the central base-station with the aid of realistically modelled imperfect optical-fiber links. When a Mobile Station (MS) is located at the cell-edge, the two nearest RAs can be invoked to detect and forward the user's signal to the base-station, based on the Single-Input Multiple-Output (SIMO) principle. Furthermore, we design a Turbo Coded (TC) 16-level Star-Quadrature Amplitude Modulation (StQAM) scheme for supporting optical-fiber-aided cooperative wireless transmission, where the receiver does not have to estimate the channel state information. Hence, a lower detection complexity can be achieved when compared to coherently detected schemes, albeit naturally, at a $3 \mathrm{~dB}$ power-loss. We also investigated the effect of phase-rotation imposed by imperfect optical-fiber links. We found that our noncoherent TC-StQAM scheme is robust to both wireless and optical-fiber imperfections.
\end{abstract}

Index Terms-Wireless communications, Optical-fiber communications, coherent, non-coherent, Turbo Code, Star-QAM, Imperfect optical fiber.

\section{INTRODUCTION}

The demand for high data rate wireless services has rapidly increased throughout the development of the mobile communications. The services facilitated by wired networks are now migrating to the wireless domain and are generally characterized by high Quality of Service (QoS) requirements. However, the cost of upgrading the infrastructure is massive. In the wired domain, optical fibers play a vital role in delivering high-rate data services. The family of hybrid techniques, which rely on a sophisticated combination of wireless mobile systems and optical fiber links have received

The financial support of European Union's Seventh Framework Programme (FP7/2007-2013) under the auspices of the CONCERTO project (grant agreement no 288502) as well as of the RC-UK under the India-UK Advanced technology Centre and the China-UK project in $4 \mathrm{G}$ wireless communications is gratefully acknowledged. substantial attention [1]. For example, Digital Fiber Optic (DFO) links [2] and Radio-over-Fiber (RoF) techniques [3] have been developed for supporting multicell systems.

The radical Unity Frequency Reuse (UFR) technique has been proposed for improving the attainable area spectral efficiency of multicell systems. However, UFR based schemes exhibit a lower cell-edge throughput due to their increased co-channel interference. Recently, the Fractional Frequency Reuse (FFR) technique has been adopted in the Third Generation Partnership Project's (3GPP) Long-Term Evolution (LTE) standard [4] as well as in the Wordwide interoperability for Microwave Access (WiMAX) system [5], since it is capable of improving the cell-edge throughput. The downlink performance of the FFR based cooperative wireless and Digital-Fiber-Optic (DFO) aided communication has been investigated in [2], where a good cell-edge performance was reported.

Diversity provides an effective mechanism of combating the effects of channel fading in wireless communication systems [6]. Single-Input MultipleOutput (SIMO) aided techniques are capable of increasing the achievable performance gain, when communicating over wireless fading channels, provided that the Channel State Information (CSI) is known at the receivers [7]-[10]. Most previous work on SIMO systems was based on the assumption of perfectly knowing the CSI and hence the corresponding data recovery typically relied on coherent detection. However, in practice it is hard to acquire accurate CSI, especially in rapidly fading mobile environments. Hence, it is of prime significance to design new non-coherent detection techniques, which dispense with channel estimation. More specifically, the soft-decision based low-complexity Star-QAM (StQAM) technique has been proposed in [11], which outperformed its hard-decision based StQAM counterpart [12], when powerful channel coding schemes [13] are invoked.

Our novel contributions in this paper may be 


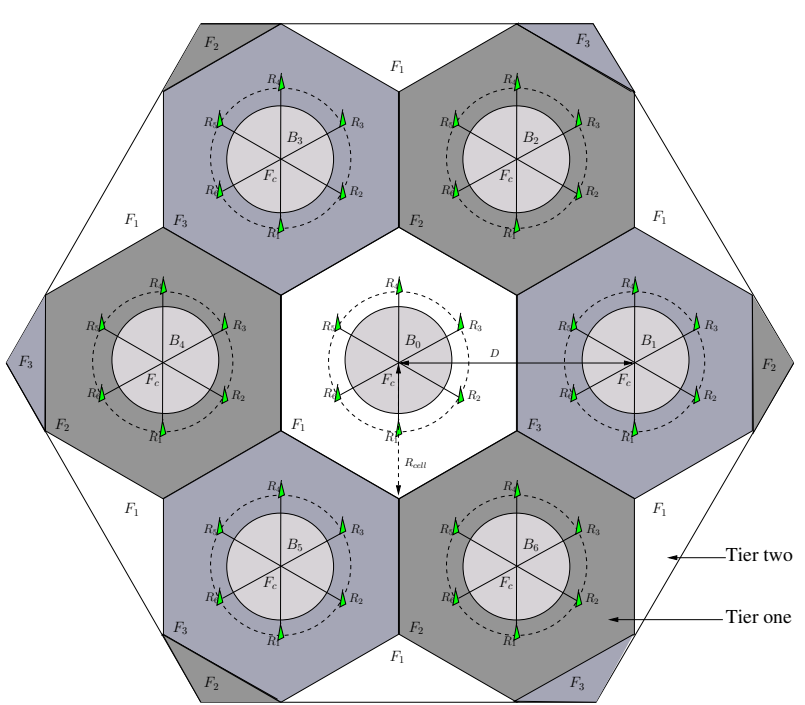

Fig. 1. Topology of the 19-cell two-tier FFR based arrangement, where $N=6$ RAs are employed in each cell.

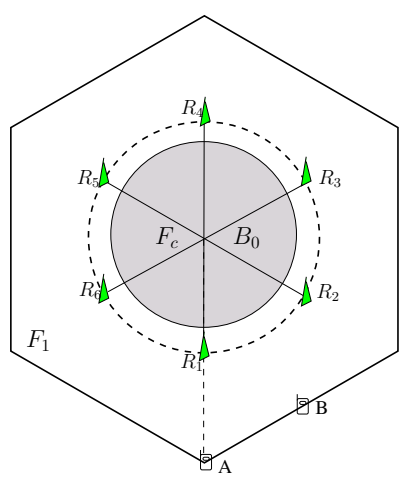

Fig. 2. The structure of the central cell and the MS considered locations $\mathrm{A}$ and $\mathrm{B}$.

summarised as follows:

1) We investigated the uplink performance of FFR based multicell multiuser schemes, as a complement of the corresponding downlink investigation in [2].

2) We further extended the Single-Input SingleOutput (SISO) based non-coherent 16StQAM scheme of [11] to a SIMO system.

3) We investigated both the Turbo-Coded [13] 16StQAM and 16QAM [12] schemes in the multicell multiuser uplink system considered, when imperfect DFO links are considered.

The outline of the paper is as follows. Our system model is described in Section II, while our results and discussions are detailed in Section III. Our conclusions are presented in Section IV.

\section{System Model AND ANALYSis}

The topology of the FFR based multicell multiuser scheme is shown in Fig. 1, where we have two tiers

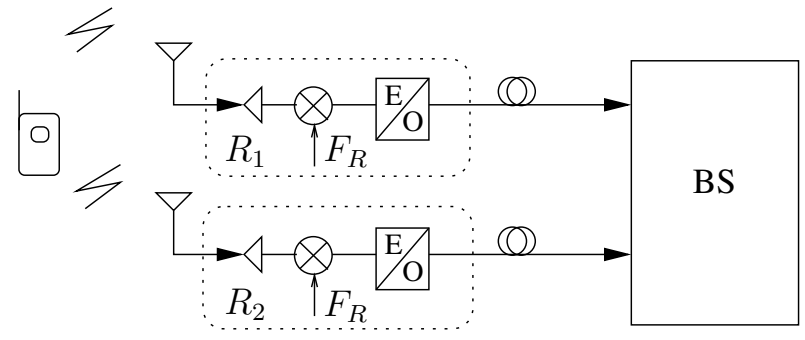

Fig. 3. The simplified system block diagram of our proposed uplink wireless-optical SIMO system, where $F_{R}$ is the radio frequency carrier and $R_{i}$ denotes the $i$ th RA.

of 19 hexagonal cells, surrounding the central BaseStation (BS) $B_{0}$ located at the origin. The frequencypartitioning strategy of the total available bandwidth $F$ is characterized by $F_{c} \bigcap F_{e}=\oslash$, where $F_{c}$ and $F_{e}$ represent the cell center's frequency band and the cell edge's frequency band, respectively. Furthermore, $F_{e}$ is divided into three orthogonal frequency bands $F_{i}$, for $i \in\{1,2,3\}$. In Fig. 1, $D$ denotes the distance between two adjacent BSs, while $R_{\text {cell }}=D / \sqrt{3}$ is the radius of each hexagonal cell. We employ $D=3 \mathrm{~km}$ for the Urban-Macro propagation scenario of [2]. We will specifically investigate the performance of uplink transmission, when the Mobile Station (MS) is located at positions $\mathrm{A}$ and $\mathrm{B}$ of Fig. 2, because these constitute the worst-case scenario at the cell-edge. The MS will be served by the nearest RA or the nearest two RAs, in the SISO or SIMO scenario, respectively.

\section{A. Imperfect Optical Fiber Model}

We assume that the links spanning from the RAs to the BS $B_{0}$, as shown in Fig. 2 and Fig. 3, are constituted by realistic rather than perfect optical fiber links. The simplest form of the Nonlinear Schrödinger (NLS) equation can be formulated as [2], [14], [15]:

$$
\frac{\partial A(z, t)}{\partial z}=(\underbrace{-i \frac{\beta_{2}}{2} \frac{\partial^{2}}{\partial t^{2}}}_{\widehat{D}}+\underbrace{i \xi|A|^{2}}_{\widehat{N}}) A(z, t),
$$

where the terms $\widehat{D}$ and $\widehat{N}$ are the simplified forms of the dispersion and the nonlinear effects, respectively. Additionally, $A(z, t)$ is a slowly varying envelope function associated with the optical pulse in the optical-fiber, where $z$ is the propagation distance and $t=t^{\prime}-z / v_{g}{ }^{1}$ is the time, while $\beta_{2}$ is the second-order propagation constant and the coefficient $\xi$ characterizes the nonlinear effects. However, the NLS equation in (1) cannot be readily solved analytically, when both linear dispersion and various optical transmission-induced nonlinear effects are present. The split-step Fourier method (SSFM) [14], [15] is one of the

${ }^{1} t^{\prime}$ is the physical time, while $v_{g}$ is the group velocity at the center wavelength. 
popular numerical algorithms used for solving the NLS equations, owing to its accuracy and relatively modest computational cost. In the so-called symmetric split-step scheme, the solution of Eq. (1) may be approximated as:

$$
\begin{aligned}
A(z+l, T) \approx & \exp \left(\frac{l}{2} \widehat{D}\right) \cdot \exp \left[\frac{l}{2}(\widehat{N}(z)+\widehat{N}(z+l))\right] \\
& \cdot \exp \left(\frac{h}{2} \widehat{D}\right) A(z, T) .
\end{aligned}
$$

Since the linear dispersion and the nonlinear operators do not commute in general, the solution given in Eq. (2) constitutes an approximation of the exact solution. The entire fiber length of $L=m l$ may be decomposed into $m$ consecutive $l$-length elements. The total optical fiber attenuation, computed based on Eq. (2), is denoted by $A_{L}$ for simplicity.

Additionally, we have also investigated the phaserotation imposed by an imperfect optical fiber. Specifically, the phase of the $k$ th optical pulse may be modelled by:

$$
\theta_{k}=\theta_{k-1}+\tilde{\delta}
$$

where $\tilde{\delta} \in[-\delta, \delta]$ is the phase difference between the $k$ th and the $(k-1)$ st optical pulse, while $\delta$ is the maximum phase difference considered.

\section{B. Detection Model}

During the first step of the transmission scenario of Fig. 3, the MS transmits its information to both the RAs $R_{1}$ and $R_{2}$. The $k$ th symbol received at the $i$ th RA $R_{i}$ may be expressed as:

$$
y_{s r_{i}, k}=\sqrt{\mathcal{L}_{s r_{i}}} h_{s r_{i}, k} x_{s, k}+n_{s r_{i}, k},
$$

where $k \in\{1, \ldots, K\}$ and $K$ is the number of symbols transmitted from the MS, while $h_{s r_{i}, k}$ denotes the Rayleigh fading coefficient between the MS and $R_{i}$. Furthermore, $n_{s r_{i}, k}$ represents the Additive While Gaussian Noise (AWGN) having a variance of $N_{0} / 2$ per dimension. Note that $\mathcal{L}_{s r_{i}}=10^{-L_{s r_{i}} / 10}$ denotes the path-loss factor between the source (MS) and the $i$ th RA $R_{i}$, where $L_{s r_{i}}=10 \aleph \log _{10}\left[\frac{d_{s r_{i}}}{d_{0}}\right]$ [16] is the pathloss attenuation in decibel, while $\aleph$ is the pathloss exponent, $d_{s r_{i}}$ is the distance between the MS, while $R_{i}$ and $d_{0}$ is the reference distance for the antenna far-field. We have considered $\aleph=2$ and $d_{0}=1 \mathrm{~km}$ in this study.

Then, the signals received from $R_{i}$ are communicated to the BS on a symbol-by-symbol basis, without demodulation or decoding. More specifically, the signal received at the $\mathrm{BS}$ from $R_{i}$ could be expressed as:

$$
\begin{aligned}
y_{i, k} & =A_{L} e^{j \theta_{k}} y_{s r_{i}, k}+n_{f}, \\
& =A_{L} e^{j \theta_{k}} \sqrt{\mathcal{L}_{s r_{i}}} x_{s, k} h_{s r_{i}, k}+A_{L} e^{j \theta_{k}} n_{s r_{i}, k}+n_{f},
\end{aligned}
$$

where again $A_{L}$ is the optical fiber's amplitude attenuation, $\theta_{k}$ is the phase-rotation introduced by the imperfect optical fiber, $n_{s r_{i}, k}$ is the noise at $R_{i}$ and $n_{f}$ is Gaussian-distributed noise with a zero mean for the optical link between $R_{i}$ and the BS. We considered $N_{r}=2$ RAs in our SIMO scheme. By denoting the $\left(1 \times N_{r}\right)$-element received signal vector by $\mathbf{Y}_{k}$, the $\left(1 \times N_{r}\right)$-element Rayleigh fading channel vector by $\mathbf{H}_{k}$ and the total $\left(1 \times N_{r}\right)$-element AWGN vector by $\mathbf{N}_{k}$, the signal received by the $\mathrm{BS}$ may be expressed as:

$$
\mathbf{Y}_{k}=A_{L} e^{j \theta_{k}} x_{s, k} \mathbf{H}_{k}+\mathbf{N}_{k}
$$

where $N_{r}=2$.

At the BS, our soft-decision aided 16StQAM modem [11] is used, which dispenses with channel estimation for the sake of low-complexity detection. The corresponding probability density function (pdf) of receiving $y_{i, k}$, when an 8PSK symbol $w_{k}$ and the amplitude-selection bit $b_{a}$ were transmitted may be written as:

$$
\begin{aligned}
& P\left(y_{i, k} \mid w_{k}, b_{a}=0\right)=\frac{1}{\pi N_{0}^{(0)}} e^{\frac{-\left|y_{i, k}-y_{i, k-1} \alpha^{(0)} w_{k}\right|^{2}}{N_{0}^{(0)}}} \\
& P\left(y_{i, k} \mid w_{k}, b_{a}=1\right)=\frac{1}{\pi N_{0}^{(1)}} e^{\frac{-\left|y_{i, k}-y_{i, k-1} \alpha^{(1)} w_{k}\right|^{2}}{N_{0}^{(1)}}}+ \\
& \frac{1}{\pi N_{0}^{(2)}} e^{\frac{-\left|y_{i, k}-y_{i, k-1}{ }^{(2)} w_{k}\right|^{2}}{N_{0}^{(2)}}}
\end{aligned}
$$

where $b_{a}$ is the bit used for the selection of the two possible amplitudes, namely $a^{(1)}$ and $a^{(2)}$, while the amplitude ratio $\alpha^{(i)}$ may be expressed as:

$$
\alpha^{(i)}=\left\{\begin{array}{lrl}
\frac{a^{(1)}}{a^{(1)}} \text { or } \frac{a^{(2)}}{a^{(2)}}=1 ; & i=0 \\
\frac{a^{(1)}}{a^{(2)}} ; & i=1 \\
\frac{a^{(2)}}{a^{(1)}} ; & i=2 .
\end{array}\right.
$$

The effective noise variance related to the noise at $R_{i}$ and BS, $n_{i, k}$, depends on the amplitude ratio $\alpha_{i}$ used at the $k$ th time instant, which may be estimated as:

$$
\begin{aligned}
N_{0}^{(i)} & =N_{f}+\left|\alpha^{(i)}\right|^{2}\left|A_{L}\right|^{2}\left|w_{k}\right|^{2} N_{0}, \\
& =N_{f}+\left|\alpha^{(i)}\right|^{2}\left|A_{L}\right|^{2} N_{0},
\end{aligned}
$$

where the amplitude of an 8PSK symbol is unity, $\left|w_{k}\right|=1$, while the noise at the $\mathrm{BS} n_{f}$ represents the AWGN having a variance of $N_{f} / 2$ per dimension. Finally, the pdf of receiving $y_{1, k}$ and $y_{2, k}$ conditioned on the transmission of $\left\{b_{0}, b_{1}, b_{2}\right\}$ (the three bits mapped to an 8PSK symbol) and $b_{a}$ can be computed as:

$$
\begin{aligned}
P\left(y_{1, k}, y_{2, k} \mid b_{0}, b_{1}, b_{2}, b_{a}\right)= & P\left(y_{1, k} \mid w_{k}, b_{a}\right) \times \\
& P\left(y_{2, k} \mid w_{k}, b_{a}\right),(
\end{aligned}
$$

which is then fed to the turbo decoder [13] for generating the original information bit sequence transmitted by the mobile user. 


\section{Simulation Results}

In this section, we characterize the proposed system of Fig. 2, where the simulation parameters are summarized in Table I. The Turbo-Coded 16QAM (TC-16QAM) scheme is used in this section as the benchmark.

\begin{tabular}{|l|r|}
\hline Modulation & $\begin{array}{r}\text { 16StQAM, } \\
\text { 16QAM }\end{array}$ \\
\hline Mapping & Set Partitioning (SP) [12] \\
\hline Coding & TC \\
\hline $\begin{array}{l}\text { Constituent } \\
\text { Code }\end{array}$ & $\left.\begin{array}{r}\text { Half-rate Recursive Systematic Convolutional (RSC) code } \\
\text { Code Polynomial G=[15 }\end{array}\right]$ \\
\hline $\begin{array}{l}\text { Code } \\
\text { Memory }\end{array}$ & 3 \\
\hline $\begin{array}{l}\text { Outer iter- } \\
\text { ations }\end{array}$ & 1 \\
\hline $\begin{array}{l}\text { Inner TC } \\
\text { iterations }\end{array}$ & 4 \\
\hline Decoder & Approximate Log-MAP \\
\hline $\begin{array}{l}\text { Symbols } \\
\text { per block }\end{array}$ & 1200 \\
\hline $\begin{array}{l}\text { Number of } \\
\text { frames }\end{array}$ & 5 000 \\
\hline Channel & Correlated Rayleigh fading channel \\
\multicolumn{2}{|c|}{ Optical channel } \\
\hline
\end{tabular}

TABLE I

SIMULATION PARAMETERS.

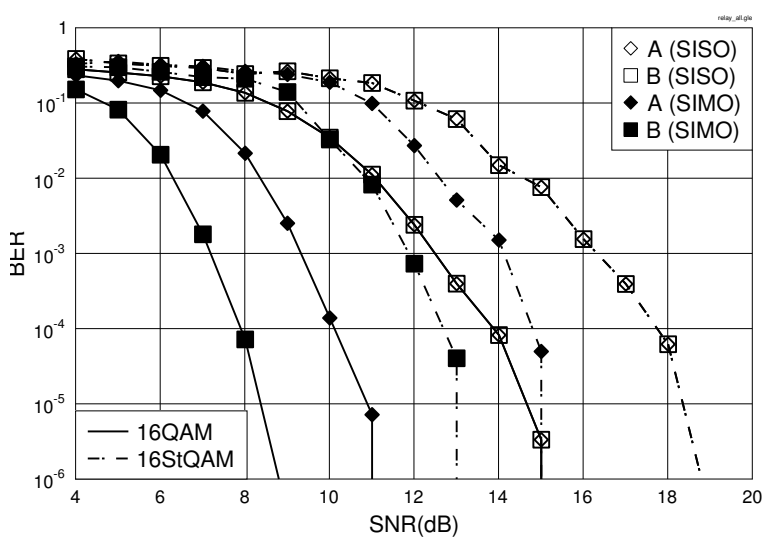

Fig. 4. BER versus SNR performance of the TC-16QAM and TC-16StQAM schemes when transmitting over correlated Rayleigh fading wireless and imperfect optical channels. The simulation parameters are summarised in Table I and the corresponding cell is shown in Fig. 2.

Fig. 4 denotes the Bit Error Ratio (BER) versus SNR performance of both the TC-16QAM and TC16StQAM schemes, when transmitting over correlated Rayleigh fading and imperfect optical channels. As seen in Fig. 4, the performance at locations A and B is identical for the SISO scenario, because they have an identical transmission distance of $d_{s r_{i}}=\sqrt{3} / 2 \mathrm{~km}$ from the nearest RA, according to Fig. 2. The TC16QAM-SISO scheme outperforms the TC-16StQAMSISO scheme by approximately $4 \mathrm{~dB}$ at a BER of $10^{-6}$, when a perfect CSI is available at the BS. When the SIMO scheme is considered, the performance at location $\mathrm{B}$ improves more significantly than that at location A. This is because location B has the same distance from $R_{1}$ and $R_{2}$ according to Fig. 2, while location $\mathrm{A}$ is further away from $R_{2}$. More specifically, the TC-16QAM-SIMO scheme performs approximately $6 \mathrm{~dB}$ better than the TC-16QAM-SISO scheme at a BER of $10^{-6}$.

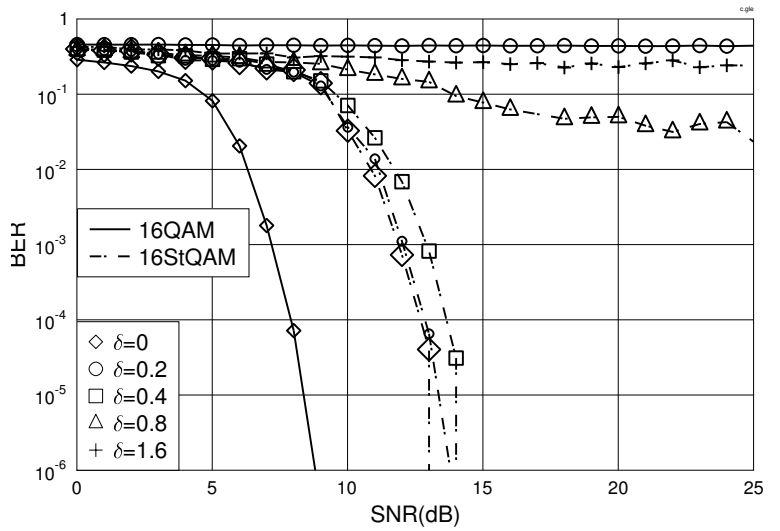

Fig. 5. BER versus SNR performance of the TC-16QAMSIMO and 16StQAM-TC-SIMO schemes of Fig. 2 and Fig. 3 for transmission over correlated Rayleigh fading and imperfect optical channels. The corresponding simulation parameters are summarised in Table I. Phase rotations of upto $\delta=\{0,0.2,0.4,0.8,1.6\}$ radian are considered in the optical link.

However, the 16QAM-based scheme cannot work well, when phase-rotations are imposed by the imperfect optical-fiber. More specifically, Fig. 5 shows the performance of both the TC-16QAM-SIMO and TC16StQAM-SIMO schemes, when phase rotations of upto $\delta=\{0,0.2,0.4,0.8,1.6\}$ radian are considered in the optical link. We assume that the CSI of the wireless link is available at the BS for the TC-16QAM-SIMO, but the phase-rotation inflicted by the optical-fiber link is unknown. As seen from Fig. 5, the TC-16QAMSIMO scheme breaks down at $\delta=0.2$ radian, while the proposed TC-16StQAM-SIMO can still perform adequately even for $\delta=0.4$ radian.

Let us investigate the BER performance at the celledge scenario of Fig. 2 in our FFR-based cellular system. The 3D BER versus SNR performance of the TC-16StQAM-SISO scheme is shown in Fig. 6 for SNR $=5 \mathrm{~dB}$. There are 12 BER peaks in Fig. 6, which correspond to all the $12 \mathrm{~A}$ and $\mathrm{B}$ locations (each $60^{\circ}$ sector has one location $\mathrm{A}$ and one location $\mathrm{B}$ ) in the hexagonal cell.

When two RAs are used for detecting the mobile user's signals, the 3D BER performance of the TC16StQAM-SIMO scheme is depicted in Fig. 7 for SNR $=5 \mathrm{~dB}$. The six BER peaks corresponding to the six locations of $\mathrm{B}$ have disappeared, as a benefit of the SIMO scheme. The six BER peaks corresponding to the six A locations have been reduced, although not completely removed. The BER surface seen in Fig. 7 exhibits a more wide-spread low-BER area compared to that seen in Fig. 6. This indicates that a better uplink transmission quality can be achieved, when our SIMO 
scheme is invoked.

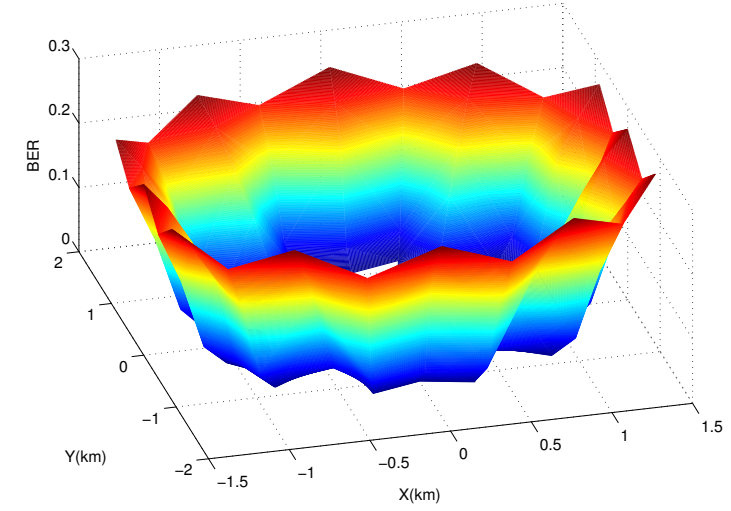

Fig. 6. The 3D BER versus SNR performance of the TC-16StQAMSISO scheme of Fig. 2 for transmission over correlated Rayleigh fading wireless and imperfect optical channels for the whole cell with $\mathrm{SNR}=5 \mathrm{~dB}$. A phase rotation of upto $\delta=0.1$ radian is considered in the optical link. The other simulation parameters are summarised in Table I.

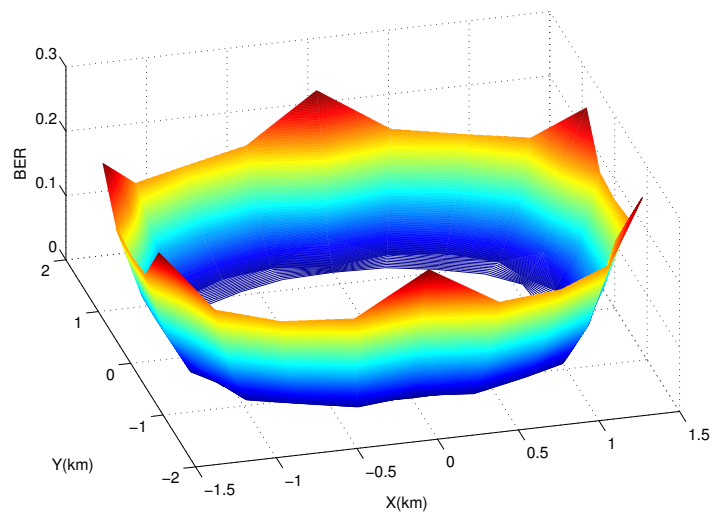

Fig. 7. The 3D BER versus SNR performance of the 16StQAMTC-SIMO scheme of Fig. 2 for transmission over correlated Rayleigh fading wireless and imperfect optical channels for the whole cell with $\mathrm{SNR}=5 \mathrm{~dB}$. A phase rotation of upto $\delta=0.1$ radian is considered in the optical link. The corresponding simulation parameters are summarised in Table I.

\section{CONCLUSIONS}

In this contribution, we have investigated the uplink performance of FFR based multicell, multiuser schemes, where cooperative wireless and optical-fiber communications were combined. We have derived the SIMO-based soft-demodulation for StQAM and found that the proposed TC-StQAM scheme is robust to both wireless and optical-fiber induced channel impairments. More explicitly, the proposed TC-StQAM-SIMO scheme is capable of removing 6 out of 12 BER peaks at the cell-edge, despite dispensing with CSI for both the wireless and optical-fiber links. The proposed TCStQAM-SIMO scheme constitutes an attractive lowcomplexity detection candidate for FFR based multicell, multiuser LTE and WiMAX systems.

\section{REFERENCES}

[1] G. Kardaras, T. T. Pham, J. Soler, and L. Dittmann, "Analysis of control and management plane for hybrid fiber radio architectures," in 2010 12th IEEE International Conference on Communication Technology (ICCT), Nov. 2010, pp. $281-284$.

[2] X. Xu, R. Zhang, S. Ghafoor, and L. Hanzo, "Imperfect digital-fiber-optic-link-based cooperative distributed antennas with fractional frequency reuse in multicell multiuser networks," IEEE Transactions on Vehicular Technology, vol. 60, no. 9, pp. 4439 -4449, Nov. 2011.

[3] D. Wake, M. Webster, G. Wimpenny, K. Beacham, and L. Crawford, "Radio over fiber for mobile communications," in IEEE International Topical Meeting on Microwave Photonics, Oct. 2004, pp. $157-160$.

[4] H. Ekstrom, A. Furuskar, J. Karlsson, and et al, "Technical solutions for the $3 \mathrm{G}$ long-term evolution," IEEE Communications Magazine, vol. 44, pp. 38- 45, Mar. 2006.

[5] A. Ghosh, D. Wolter, J. Andrews, and R. Chen, "Broadband wireless access with WiMax/802.16: current performance benchmarks and future potential," IEEE Communications Magazine, vol. 43, pp. 129-136, Feb. 2005.

[6] L. Hanzo, O. Alamri, M. El-Hajjar and N. Wu, Near-Capacity Multi Functional MIMO Systems. Wiley-IEEE Press, May 2009.

[7] K. J. Ray Liu, A. K. Sadek, W. Su, A. Kwasinski, Cooperative Communications and Networking. Cambridge University Press, Dec. 2008.

[8] T. Cover and A. Gamal, "Capacity theorems for the relay channel," IEEE Transactions on Information Theory, vol. 25 , no. 5, pp. 572 - 584, Sep. 1979.

[9] A. Sendonaris, E. Erkip, and B. Aazhang, "User cooperation diversity. Part I. System description," IEEE Transactions on Communications, vol. 51, no. 11, pp. 1927 - 1938, Nov. 2003.

[10] - "User cooperation diversity. Part II. Implementation aspects and performance analysis," IEEE Transactions on Communications, vol. 51, no. 11, pp. 1939 - 1948, Nov. 2003.

[11] D. Liang, S. X. Ng, and L. Hanzo, "Soft-decision Star-QAM aided BICM-ID," IEEE Signal Processing Letters, vol. 18 , no. 3, pp. $169-172$, March 2011.

[12] L. Hanzo, S. X. Ng, T. Keller, and W. Webb, Quadrature Amplitude Modulation: From Basics to Adaptive Trellis-Coded, Turbo-Equalised and Space-Time Coded OFDM, CDMA and MC-CDMA Systems Digital Communications, 2nd ed. WileyIEEE Press, 2004.

[13] L. Hanzo, T. H. Liew, B. L. Yeap, R. Y. S. Tee and S. $\mathrm{X}$. Ng, Turbo Coding, Turbo Equalisation and Space-Time Coding: EXIT-Chart-Aided Near-Capacity Designs for Wireless Channels. Wiley-IEEE Press, 2011.

[14] J.K.Shaw, Mathematical Principles of Optical Fiber Cоттиnications. Society for Industrial and Applied Mathematics, 2004.

[15] G. P. Agrawal, Nonlinear fiber optics, 4th ed. Academic Press, 2006.

[16] A. Goldsmith, Wireless Communications. Cambridge University Press, 2005 